La Revue

des Droits

de l'Homme

\section{La Revue des droits de l'homme}

Revue du Centre de recherches et d'études sur les droits fondamentaux

Actualités Droits-Libertés | 2020

\title{
Annulation du retrait de DACA aux États-Unis : une décision aux effets contrastés
}

Note sous Cour suprême des États-Unis, Department of Homeland

Security v. Regents of the University of California, 591 U.S. _- (2020).

\section{Léa Boinnard}

\section{(2) OpenEdition}

\section{Journals}

Electronic version

URL: http://journals.openedition.org/revdh/10457

DOI: $10.4000 /$ revdh. 10457

ISSN: 2264-119X

\section{Publisher}

Centre de recherches et d'études sur les droits fondamentaux

\section{Electronic reference}

Léa Boinnard, «Annulation du retrait de DACA aux États-Unis : une décision aux effets contrastés », La Revue des droits de l'homme [Online], Actualités Droits-Libertés, Online since 27 September 2020, connection on 06 November 2020. URL : http://journals.openedition.org/revdh/10457 ; DOI : https:// doi.org/10.4000/revdh. 10457

This text was automatically generated on 6 November 2020 .

Tous droits réservés 


\section{Annulation du retrait de DACA aux États-Unis : une décision aux effets contrastés}

Note sous Cour suprême des États-Unis, Department of Homeland

Security v. Regents of the University of California, 591 U.S. _- (2020).

\section{Léa Boinnard}

1 Alors que le Président américain, Donald Trump, a centré une part importante de sa campagne et de sa politique sur la lutte contre l'immigration irrégulière, la Cour suprême, à majorité conservatrice ${ }^{1}$, vient de faire prudemment obstacle à ses ambitions. Dans une décision rendue à cinq voix contre quatre, celle-ci a en effet jugé que le retrait par l'exécutif d'un programme adopté par l'administration Obama, DACA (Deferred Action for Childhood Arrival), était illégal ${ }^{2}$. L'opinion majoritaire, rédigée par le président conservateur de la Cour, John Roberts, et soutenue par les quatre juges à tendance plus libérale (Ruth Bader Ginsburg, Stephen Breyer, Sonia Sotomayor et Elena Kagan), a pu surprendre en raison de son caractère favorable aux Dreamers, expression désignant les étrangers en situation irrégulière arrivés enfants aux États-Unis, visés par le programme DACA. Les juges conservateurs, Clarence Thomas (soutenu par Samuel Alito et Neil Gorsuch), Samuel Alito et Brett Kavanaugh, ont quant à eux proposé des opinions en partie dissidentes, soutenant la légalité du retrait. Si la décision représente a priori une avancée pour les droits des étrangers en situation irrégulière aux ÉtatsUnis, elle n'en reste pas moins limitée dans sa portée.

2 L'arrêt de la Cour suprême américaine dans l'affaire Department of Homeland Security $v$. Regents of the University of California s'inscrit dans un contentieux plus vaste au sujet de deux programmes - DACA et DAPA (Deferred Action for Parents of Americans) - adoptés par l'administration Obama en matière d'immigration. Le premier, créé en juin 2012 par un mémorandum ${ }^{3}$, vise à protéger de l'éloignement certains étrangers en situation irrégulière, c'est-à-dire ne disposant d'aucun droit au séjour sur le territoire. Les personnes y sont éligibles lorsqu'elles sont arrivées aux États-Unis pendant leur enfance, et répondent à différents critères, notamment d'âge (des jeunes adultes), de 
diplômes ou d'engagement dans l'armée américaine, et d'absence de condamnation pénale. Un statut temporaire leur est alors accordé, leur permettant de travailler, mais ne constituant pas pour autant un titre de séjour ni une voie vers la citoyenneté. En novembre 2014, par un second mémorandum, l'exécutif avait élargi la catégorie des personnes éligibles à DACA et étendu ces mesures aux parents d'enfants citoyens américains ou résidents permanents, avec l'adoption du programme DAPA ${ }^{4}$.

Le mémorandum de 2014 avait fait l'objet d'une première affaire judiciaire, déclenchée par un recours du Texas et de 25 autres États américains. Les juridictions fédérales de première instance et d'appel avaient accepté les recours, considérant que DAPA faisait obstacle à une évaluation suffisamment individualisée des demandes, et avait été adopté en méconnaissant les règles de procédure administrative ${ }^{5}$. Selon la décision du tribunal de première instance ${ }^{6}$, l'administration avait manqué à ses obligations de consultation du public et de recueil de commentaires prévues par la loi sur la procédure administrative (Administrative Procedure Act - APA), rendant la décision illégale. La cour d'appel avait ajouté à ce motif celui du caractère "arbitraire et capricieux» de la mesure au sens de cette même loi. Les deux juridictions avaient toutefois rejeté l'argument des États selon lequel l'adoption du programme méconnaissait l'obligation constitutionnelle du Président d'exécuter fidèlement la loi ${ }^{7}$. Dans un jugement rendu à quatre voix contre quatre en raison de la vacance d'un siège, la Cour suprême avait maintenu la décision de la cour d'appel, sans toutefois établir de précédent, comme le prévoient les règles en la matière ${ }^{8}$.

4 En conséquence de cette décision, l'administration Trump avait annoncé le retrait du mémorandum portant extension de DACA et création de DAPA9 . Trois mois après, la secrétaire d'État à la sécurité intérieure Elaine $C$. Duke adoptait un mémorandum retirant le programme DACA ${ }^{10}$. Celui-ci se fondait sur la décision rendue par la Cour suprême dans l'arrêt United States v. Texas, bien que l'affaire ne concerne pas DACA mais son extension et DAPA, et sur une lettre du Procureur général qui jugeait le programme illégal.

5 C'est cette décision de retrait qui a fait l'objet des recours d'associations, d'étrangers en situation irrégulière et de recteurs d'université dans l'affaire Department of Homeland Security $v$. Regents of the University of California. Trois affaires, en Californie (Department of Homeland Security v. Regents of the University of California), à New York (Trump v. NAACP) et dans le District de Columbia (Wolf $v$. Vidal), avaient amené les juridictions fédérales de première instance à rendre des décisions en faveur des requérants, sur différents fondements, y compris la loi sur la procédure administrative et la clause de procédure légale régulière du cinquième amendement de la Constitution. Le gouvernement avait fait appel de ces décisions, dont l'une a été confirmée par la cour d'appel du neuvième circuit $^{11}$. Cet arrêt a ensuite fait l'objet de l'appel devant la Cour suprême, qui a joint les trois affaires.

6 À l'appui de leur requête, les requérants ont fait valoir différents moyens, en particulier la méconnaissance des règles de procédure prévues par l'APA, et la violation de la clause d'égale protection de la loi, garantie par le cinquième amendement de la Constitution américaine. Le gouvernement jugeait quant à lui le recours irrecevable, estimant qu'il s'agissait d'une action discrétionnaire du pouvoir exécutif, qui ne relevait pas du contrôle du juge. De plus, il considérait que le retrait de DACA s'imposait à lui, dès lors que le programme pouvait être analysé comme illégal au regard de la décision United States v. Texas. 
$7 \quad$ Les enjeux de la décision Department of Homeland Security v. Regents of the University of California étaient nombreux, et ses conséquences importantes pour la situation de millions d'étrangers en situation irrégulière résidant sur le territoire américain. Audelà du cas d'espèce, elle était attendue pour évaluer l'attitude de la Cour suprême face aux politiques migratoires de Donald Trump - et du pouvoir exécutif en général - dans un contexte de blocage des tentatives de réformes législatives, et de politiques de plus en plus hostiles à l'immigration. Ces évolutions amènent également à s'interroger sur la protection constitutionnelle des étrangers en situation irrégulière aux États-Unis, laquelle demeure contrastée. À ces égards, la décision a pu s'avérer décevante, car elle apporte peu de réponses.

8 Si la Cour suprême consacre une protection provisoire aux bénéficiaires de DACA sur des fondements procéduraux, elle ne tranche toutefois pas la question de la séparation des pouvoirs en matière d'immigration (I). De plus, elle n'a pas saisi l'occasion de clarifier le statut constitutionnel des étrangers en situation irrégulière, et leur apporte donc une protection limitée (II).

\section{I/- Une protection provisoire des bénéficiaires de DACA sur des fondements procéduraux}

9 Certes, la décision Department of Homeland Security v. Regent of the University of California a mis un frein à la politique de l'administration Trump en matière d'immigration, mais la protection des Dreamers n'est que provisoire. Tout en s'abstenant de trancher la question de la légalité de DACA et, par conséquent, de clarifier la séparation des pouvoirs en matière d'immigration (A), la Cour a fondé sa décision sur des manquements procéduraux au regard de la loi sur la procédure administrative (B), laissant la possibilité à l'administration de retirer le programme sur d'autres fondements.

\section{A/- Une occasion manquée de clarifier la séparation des pouvoirs en matière d'immigration}

10 En s'abstenant de trancher la question de la légalité du programme DACA en lui-même, la Cour a écarté l'opportunité qui se présentait à elle de clarifier la séparation des pouvoirs entre le législatif et l'exécutif en matière d'immigration, et ainsi maintenu le trouble laissé par l'arrêt United States v. Texas. En effet, la confirmation par la Cour suprême de l'arrêt de la cour d'appel à égalité des voix dans cette affaire avait remis en cause la légalité des programmes d'action différée (deferred action) en matière d'immigration ${ }^{12}$. Utilisée depuis longtemps par les présidents américains ${ }^{13}$, cette pratique consiste pour l'exécutif à user de son pouvoir discrétionnaire de poursuite afin d'éviter l'éloignement de certains étrangers en situation irrégulière, sans pour autant les régulariser ${ }^{14}$.

11 Alors que le Congrès dispose d'un pouvoir général en matière d'immigration ${ }^{15}$, l'action différée s'inscrivait en théorie dans le pouvoir discrétionnaire de l'exécutif en matière d'application de la loi ${ }^{16}$. En effet, la loi sur l'immigration et la nationalité (Immigration and Nationality Act - INA) confère à l'exécutif le pouvoir d'établir les politiques et de fixer les priorités d'application des lois en matière d'immigration au niveau national ${ }^{17}$. 
Son pouvoir général d'opportunité des poursuites lui permet également de donner la priorité à certains éloignements plutôt qu'à d'autres.

L'arrêt United States $v$. Texas ayant remis en cause ces fondements, sans toutefois créer de précédent, l'affaire Department of Homeland Security $v$. Regents of the University of California aurait pu être l'occasion de trancher la constitutionnalité de DACA et, par extension, d'apporter des éléments sur la légalité des programmes d'action différée en général. Malgré les appels en ce sens de la part des parties et de certains juges lors de l'audience, la Cour s'en est abstenue.

13 Une telle réponse était d'autant plus attendue que les États-Unis connaissent un blocage inédit des politiques migratoires en raison de l'absence de consensus pour une réforme globale entre le Congrès et le pouvoir exécutif ${ }^{18}$. En 2001, une proposition de loi avait été présentée devant le Congrès, sous l'acronyme de DREAM Act (pour Development, Relief, and Education for Alien Minors). Celle-ci visait à conférer un statut à des étrangers en situation irrégulière arrivés mineurs aux États-Unis, afin d'éviter qu'ils ne soient éloignés vers leur pays d'origine. L'objectif de cette réforme était d'apporter une réponse au nombre important d'étrangers résidant irrégulièrement sur le territoire (estimés à 10,7 millions en 2017 , soit $3,2 \%$ de la population américaine ${ }^{19}$ ), en favorisant la régularisation de personnes intégrées, dans un contexte où l'administration ne dispose pas de moyens suffisants pour éloigner toutes les personnes concernées.

14 Malgré un soutien bipartisan, l'acte ne fut pas adopté du fait de son rejet au Sénat. Dans les seize années qui ont suivi, le Congrès a proposé de nombreuses lois similaires, mais toutes furent refusées. Face à cet échec, l'administration Obama avait adopté DACA, dans le but de protéger temporairement ces personnes de l'éloignement, avec l'espoir que le Congrès adopte finalement le DREAM Act ${ }^{20}$.

15 En écartant la question de la légalité de DACA de sa décision, la Cour suprême ne se prononce donc pas sur l'étendue des pouvoirs du Président pour faire face à ce qui apparaît comme une carence $d u$ Congrès, les lois migratoires ne pouvant manifestement pas être exécutées dans leur état actuel. Elle fait par conséquent preuve d'une déférence relativement importante au pouvoir politique (exécutif et législatif) pour la gestion de l'immigration. Parallèlement, la Cour fait un pas en faveur de la protection des Dreamers, en annulant le retrait de DACA sur le fondement de la procédure administrative, et signifie ainsi à l'administration Trump qu'elle ne bénéficie pas d'une marge de manœuvre sans limites en la matière.

\section{B/- La procédure administrative comme limite au pouvoir exécutif}

Dans ce contexte de blocage de réforme au Congrès et d'intervention croissante du gouvernement en matière d'immigration, la Cour se fonde sur la procédure administrative pour limiter le pouvoir exécutif. Elle déclare d'abord la requête recevable et conclut au caractère « arbitraire et capricieux » du retrait.

17 En premier lieu, la Cour rejette les arguments du gouvernement selon lesquels le retrait de DACA ne relèverait pas du contrôle du juge, car la décision de l'exécutif de ne pas mettre en œuvre une loi dans un cas précis s'inscrirait dans son pouvoir discrétionnaire en la matière ${ }^{21}$. La Cour exclut cette interprétation et rappelle que cette exception de recevabilité ne concerne que des décisions individuelles. Elle estime que DACA n'est pas uniquement une politique qui permet aux autorités migratoires de ne 
pas exécuter une loi dans un cas individuel, mais qu'il confère également des bénéfices pour les personnes concernées, comme le droit au travail ou à la sécurité sociale. Dès lors, la Cour juge qu'elle peut examiner les moyens fondés sur la méconnaissance des règles de procédure administrative.

En second lieu, la Cour examine le caractère « arbitraire et capricieux » de la mesure de retrait, au sens de l'APA ${ }^{22}$. Selon l'opinion majoritaire, bien que l'administration Trump fut en droit de retirer le programme adopté par l'administration Obama, celle-ci n'a pas suffisamment justifié sa décision. En effet, le mémorandum de retrait initial, adopté par la secrétaire d'État Elain Duke, se basait exclusivement sur l'idée que DACA était illégal, ce qui ne constituait pas une justification suffisante aux yeux de la Cour. Celle-ci a par ailleurs refusé de prendre en compte un mémorandum adopté postérieurement au début de l'affaire par la nouvelle secrétaire d'État aux affaires intérieures, Kirstjen Nielsen, en réponse à l'arrêt de la Cour de district du District de Columbia ${ }^{23}$.

Ainsi, se référant uniquement au mémorandum Duke, la majorité considère que l'administration n'a pas suffisamment pris en compte les effets du retrait sur la population bénéficiant de DACA. Des intérêts essentiels en jeu, notamment l'intégration des individus dans des communautés et les activités qu'ils ont entreprises, n'ont pas été intégrés dans la décision du gouvernement, rendant cette décision «arbitraire et capricieuse ». La Cour ne juge pas pour autant que l'administration ne pourrait retirer DACA sous aucun prétexte. Elle considère seulement qu'un tel retrait devrait se faire en mettant en balance les différents intérêts en cause, lesquels étaient importants en l'espèce.

La Cour signifie ainsi implicitement au Président que celui-ci doit assumer la responsabilité politique de la décision de retrait. En effet, Donald Trump avait déclaré lui-même au début de son mandat qu'il ne comptait pas revenir sur DACA, programme relativement populaire aux États-Unis ${ }^{24}$. La Cour attache ainsi de l'importance à la forme des décisions de l'exécutif, et à l'honnêteté de leur justification face au public.

Bien que cette décision, limitant les pouvoirs exécutifs en matière d'immigration, ait pour conséquence de protéger - au moins temporairement - les étrangers bénéficiaires de DACA, elle revêt une portée relative, dès lors qu'elle est fondée sur des aspects de procédure administrative, et non sur des principes constitutionnels. Au même titre que dans la décision United States $v$. Texas, le sort de milliers de personnes est scellé par des questions de procédure, sans tenir compte de la situation humanitaire dans laquelle ceux-ci se trouvent. Or, plusieurs requérants, des juges fédéraux, mais également la juge Sotomayor dans son opinion individuelle, estimaient que la question des droits fondamentaux des bénéficiaires de DACA, notamment au regard du principe d'égale protection de la loi, devait être examinée.

\section{II/- Un concours limité à une protection constitutionnelle des étrangers en situation irrégulière}

La décision Department of Homeland Security v. Regents of the University of California offre finalement une protection limitée aux étrangers en situation irrégulière, puisqu'elle ne se fonde pas sur des dispositions constitutionnelles et relatives aux droits fondamentaux. En rejetant les moyens tirés des déclarations discriminatoires du Président Trump lors de sa campagne et de sa présidence (A), la Cour suprême s'inscrit 
dans la lignée de décisions écartant les questions de protection des droits fondamentaux des étrangers en situation irrégulière depuis plusieurs années, menant à une protection constitutionnelle peu substantielle de ces derniers (B).

\section{A/- La confirmation de l'absence de prise en compte des déclarations de l'exécutif}

En écartant les moyens tirés des déclarations faites par le Président Trump avant et depuis son élection sur la communauté latino-américaine et sur les immigrés en général, la Cour a confirmé sa position adoptée deux ans auparavant dans l'arrêt Trump v. Hawaii ${ }^{25}$. Dans cette décision, la Cour suprême avait refusé de tenir compte des déclarations de Donald Trump sur les musulmans dans son évaluation du caractère potentiellement discriminatoire des mesures d'interdiction d'entrée sur le territoire visant les ressortissants de huit pays, principalement à majorité musulmane. Cette décision avait été fortement critiquée du fait du caractère inédit de la déférence de la Cour envers le pouvoir politique en la matière.

La décision Department of Homeland Security v. Regents of the University of California adopte une position similaire puisque la majorité rejette les griefs tirés du caractère discriminatoire du retrait de DACA envers les immigrés latino-américains. Les requérants s'appuyaient en effet sur les déclarations du Président concernant la population immigrée d'origine latino-américaine, notamment mexicaine, qualifiée par exemple de «trafiquants de drogues criminels et violeurs ${ }^{26}$. La Cour aurait pu s'écarter de la position adoptée dans Trump v. Hawaii, dès lors qu'il ne s'agissait pas ici d'étrangers à l'extérieur des États-Unis mais de personnes présentes sur le territoire. Celles-ci bénéficient en principe de la protection de la clause de procédure légale régulière, même lorsqu'elles sont en situation irrégulière ${ }^{27}$.

25 La majorité rejette cependant l'idée selon laquelle le retrait de DACA serait motivé par une «animosité » envers la communauté mexicaine, ce qui le rendrait illégal car discriminatoire. Elle s'appuie pour cela sur plusieurs éléments, qui constituent un faisceau d'indices pour évaluer l'animosité d'une décision ${ }^{28}$. En premier lieu, les Mexicains représentant une part importante des étrangers en situation irrégulière aux États-Unis, elle ne juge pas discriminatoire le fait que le retrait de DACA affecte plus particulièrement cette communauté. Elle aurait pourtant pu être considérée comme « classe vulnérable sans pouvoir politique ${ }^{29}$. Par ailleurs, la Cour considère que l'étude des évènements ayant mené au retrait du programme ne démontre pas l'existence d'une discrimination. Enfin, elle estime que les propos tenus par le gouvernement ne sont pas concluants dans cette affaire, dès lors qu'ils datent d'avant le retrait du programme, et qu'ils n'ont pas été prononcés dans le contexte de celui-ci.

Ce refus réitéré montre un certain retrait de la Cour par rapport à des affaires antérieures. Par exemple, en 1982, dans l'arrêt de principe Plyler v. Doe rendu en matière de droits fondamentaux des étrangers en situation irrégulière, la Cour avait pris en compte l'animosité dans son évaluation de mesures visant à discriminer les enfants étrangers en situation irrégulière dans les frais d'inscription d'une école ${ }^{30}$. Elle avait notamment jugé qu'« imposer des frais supplémentaires aux enfants sans-papiers constitu[ait] une tentative lucrative et inefficiente d'endiguer l'immigration illégale ${ }^{31}$ ". Sur ce fondement, elle avait reconnu le bénéfice de la clause d'égale protection de la loi du quatorzième amendement à des enfants étrangers en situation irrégulière. 
Le rejet des déclarations de Donald Trump n'était d'ailleurs pas unanime puisque la juge Sotomayor, dans son opinion individuelle, a considéré que la Cour aurait dû les prendre en compte, comme elle l'avait déjà estimé dans l'affaire Trump v. Hawaii. Cela aurait permis, selon elle, d'examiner les questions de droits fondamentaux et, en particulier, d'égale protection de la loi qui étaient soulevées par les requérants. En écartant ces moyens, la Cour s'inscrit dans un mouvement de rejet croissant des considérations tirées des droits fondamentaux dans les affaires concernant le droit des étrangers.

\section{$B /-$ Le rejet réitéré des moyens tirés des droits fondamentaux des étrangers en situation irrégulière}

En refusant de tenir compte des propos du Président Trump sur les Mexicains, la Cour a également rejeté les arguments tirés d'une violation des droits fondamentaux des bénéficiaires de DACA. En effet, leur reconnaître le bénéfice du cinquième amendement et de l'égale protection de la loi aurait permis de leur reconnaître une protection constitutionnelle plus étendue. Par conséquent, la protection apportée par la décision aux étrangers en situation irrégulière demeure fragile.

En effet, la condition des étrangers en situation irrégulière au regard du droit constitutionnel aux États-Unis est particulièrement complexe. En raison de leur séjour irrégulier sur le territoire - en violation du droit de l'immigration - ils ne bénéficient pas de l'intégralité des droits dont jouissent les citoyens et les résidents permanents. Toutefois, une part importante d'entre eux est installée sur le territoire, et est souvent incitée à travailler car elle représente une main-d'œuvre bon marché. Par ailleurs, leur situation juridique et socio-économique en fait un groupe particulièrement vulnérable, sans pouvoir politique ${ }^{32}$.

30 Le retrait de DACA priverait les personnes éligibles de droits acquis, ainsi que d'une certaine sécurité juridique concernant leur statut. Bien qu'enjoignant à l'autorité administrative de prendre en compte les effets de sa décision sur cette population, la Cour ne considère pas que le retrait porterait en lui-même une atteinte constitutionnelle aux droits des personnes concernées. Or, comme le recommandait la juge Sotomayor dans son opinion individuelle, la Cour aurait pu entendre les requérants sur ces questions et évaluer si le retrait de droits à des personnes ayant des liens forts avec les États-Unis et, parfois, faibles avec leur pays d'origine, n'entrait pas en contradiction avec leur droit à une égale protection de la loi. Des craintes existent également quant à l'usage des données que les bénéficiaires avaient fournies pour bénéficier de DACA à des fins d'éloignement, en cas de retrait du programme ${ }^{33}$.

Ce silence sur la protection constitutionnelle des bénéficiaires de DACA s'inscrit dans une tendance présente depuis plusieurs années à la Cour suprême, qui consiste à juger les affaires concernant le droit des étrangers, en particulier en situation irrégulière, sur des fondements autres que les questions des droits fondamentaux, y compris lorsque ceux-ci sont soulevés par les parties. Ces questions concernent par exemple la répartition des compétences entre échelon fédéral et États fédérés ${ }^{34}$, la séparation des pouvoirs entre exécutif et législatif ${ }^{35}$, ou la procédure administrative dans le cas présent. Or, même lorsque ces fondements jouent en faveur d'une protection accrue des étrangers dans le cas concret, celle-ci reste plus superficielle que lorsqu'elle repose 
sur les droits fondamentaux. Cette tendance pourrait remettre en cause la protection fragile dont bénéficient ces personnes dans le pays.

En effet, depuis la décision Plyler v. Doe, la Cour n'a pas réaffirmé la protection constitutionnelle des étrangers en situation irrégulière sur le fondement des cinquième et quatorzième amendements de la Constitution. Or, cette décision elle-même est limitée puisqu'en l'espèce, il s'agissait d'enfants, et que la Cour avait en partie fondé sa décision sur le fait qu'ils n'étaient pas responsables de leur situation. De plus, elle les considérait comme une classe intermédiaire dans l'évaluation des risques de discrimination, et pas suspecte comme c'est le cas pour d'autres minorités. La Cour n'a donc pas saisi l'occasion de ce contentieux pour clarifier cette question ${ }^{36}$, ce qui est peu surprenant étant donné sa composition à majorité conservatrice.

Bien qu'il semble cohérent que l'administration puisse revenir sur un programme adopté par une administration précédente, le renforcement des politiques de lutte contre l'immigration irrégulière appelle, aux États-Unis comme en Europe, à un contrôle accru des risques de violations des droits fondamentaux des étrangers, lesquels sont garantis par le droit international ${ }^{37}$. Aux États-Unis, ce contrôle se construit essentiellement au travers de décisions de la Cour suprême interprétant la Constitution en faveur de la protection de certains groupes. En matière d'immigration irrégulière toutefois, la Cour montre souvent plus de déférence envers le pouvoir politique que dans d'autres domaines. La décision Department of Homeland Security $v$. Regents of the University of California s'inscrit, malgré son opposition à l'administration Trump, dans la poursuite de cette déférence, et n'apporte pas de garantie constitutionnelle supplémentaire aux millions d'étrangers résidant irrégulièrement sur le territoire américain. Malgré tout, la décision laisse un sursis aux bénéficiaires de DACA, et signifie au Président qu'en dépit de la majorité conservatrice à la Cour, celle-ci ne lui laisse pas le champ entièrement libre en matière d'immigration. Le sort de DACA pourrait ainsi être scellé par les élections américaines de novembre 2020, à moins que l'administration Trump ne parvienne à retirer le programme en bonne et due forme d'ici là. C'est en tout cas la volonté annoncée par le Président sur son compte Twitter ${ }^{38}$.

Cour suprême des États-Unis, Department of Homeland Security v. Regents of the University of California, 591 U.S. _- (2020).

Les Lettres « Actualités Droits-Libertés » (ADL) du CREDOF (pour s'y abonner) sont accessibles sur le site de la Revue des Droits de l'Homme (RevDH) - Contact 


\section{NOTES}

1. Les juges de la Cour suprême américaine sont généralement classés, selon leurs opinions rendues dans les décisions, comme appartenant plutôt à la branche "libérale " ou " conservatrice ». Actuellement, la Cour suprême dispose d'une majorité de cinq juges considérés comme "conservateurs", dont deux ont été nommés par Donald Trump, et de quatre juges considérés comme « libéraux ».

2. Cour suprême des États-Unis, Department of Homeland Security v. Regents of the University of California, 591 U.S. (2020).

3. U.S. Department of Homeland Security, Memorandum for exercising prosecutorial discretion with respect to individuals who came to the United States as children, 15 juin 2012. URL: https:// www.dhs.gov/xlibrary/assets/s1-exercising-prosecutorial-discretion-individuals-who-came-tous-as-children.pdf

4. U.S. Department of Homeland Security, Exercising prosecutorial discretion with respect to individuals who came to the United States as children and with respect to certain individuals who are the parents of U.S. citizens or permanent residents, 20 nov. 2014. URL : https://www.dhs.gov/sites/ default/files/publications/14_1120_memo_deferred_action_1.pdf.

5. Cour d'appel du cinquième circuit, 9 nov. 2015, Texas v. U.S., 809 F.3d 134.

6. Cour de district du Texas, 16 fév. 2015, Texas v. U.S., 86 F.Supp.3d 591.

7. Constitution américaine, art. $2, \$ 3$.

8. Cour suprême des États-Unis, United States v. Texas, 579 U.S.

(2016).

9. Department of Homeland Security, Rescission of memorandum providing for deferred action for parents of Americans and lawful permanent residents ("DAPA"), 15 juin 2017. URL : https:// www.dhs.gov/news/2017/06/15/rescission-memorandum-providing-deferred-action-parentsamericans-and-lawful.

10. U.S. Department of Homeland Security, Memorandum on Rescission Of Deferred Action For Childhood Arrivals (DACA), 5 sept. 2017. URL : https://www.dhs.gov/news/2017/09/05/ memorandum-rescission-daca.

11. Cour d'appel du neuvième circuit, 8 nov. 2018, Regents of the University of California $v$. Department of Homeland Security, 908F.3d 476.

12. Voir notamment la lettre de 100 juristes américains adressée au Président Trump sur le retrait de DACA. URL: https://pennstatelaw.psu.edu/sites/default/files/documents/pdfs/ Immigrants/LawProfLetterDACAFinal8.13.pdf.

13. George TENREIRO, "What the DACA2 and DAPA programs say about executive immigration reforms moving forward », New Jersey Lawyer, the Magazine, vol. 2017, nº 304, 2017, p. 85.

14. Ibid.

15. Cour suprême des États-Unis, Chae Chan Ping v. United States, 130 U.S. 581 (1889).

16. Voir Cour suprême des États-Unis, Youngstown Sheet \& Tube Company v. Sawyer, 343 U.S. 579 (1952).

17. INA, 6 U.S.C. $\$ 1103(\mathrm{a})$.

18. Lauren GILBERT, «Obama's Ruby Slippers: Enforcement discretion in the absence of immigration reform », West Virginia Law Review, vol. 116, $\mathrm{n}^{\circ}$ 1, 2013, p. 267-269.

19. Jeans Manuel KROGSTAD, « 5 facts about illegal immigration in the U.S. », Pew Research Center [en ligne], 12 juin 2019. URL: https://www.pewresearch.org/fact-tank/2019/06/12/5-factsabout-illegal-immigration-in-the-u-s/, consulté le 30 juin 2020.

20. Catherine Y. KIM, «Immigration separation of powers and the president's power to preempt ", Notre Dame Law Review, vol. 90, nº 2, 2014, p. 692.

21. Conformément à l'arrêt Heckler v. Chaney, 470 U.S. 821 (1993). 
22. APA, 5 U.S. Code, $\$ 706$ (2) (A).

23. Cour de district du District de Columbia, 24 avr. 2018, NAACP v. Trump, 298 F. Supp. 3d.

24. Voir le tweet de Donald J. Trump du 14 sept. 2017.

25. Cour suprême des États-Unis, Trump v. Hawaii, 585 U.S.

(2018).

26. Katie REILLY, « Here are all the times Donald Trump insulted Mexico », The Time, 21 août 2016. URL : https://time.com/4473972/donald-trump-\&/, consulté le 21 juill. 2020.

27. Cour suprême des États-Unis, Plyler v. Doe, 457 U.S. 202 (1982).

28. Megan L. MALLAMAS, «American animus: Where Trump v. Hawaii leaves the animus doctrine today ", Campbell Law Review, vol. 42, nº 1, 2020, p. 142.

29. Voir en ce sens, sur l'animosité envers une classe vulnérable sans pouvoir politique : Cour suprême des États-Unis, Cleburne v. Cleburne Living Ctr., 473 U.S. 432 (1985).

30. Cour suprême des États-Unis, Plyler v. Doe, 457 U.S. 202 (1982).

31. Megan L. MALlamas, «American animus: Where Trump v. Hawaii leaves the animus doctrine today », op. cit., p. 143.

32. Voir en ce sens : Jorge A. BUSTAMANTE, «Extreme vulnerability of migrants: The cases of the United States and Mexico », Georgetown Immigration Law Journal, vol. 24, $\mathrm{n}^{\circ} 3$ et 4, p. 565-584.

33. Julie RHEINSTROM, «Deferred dreams denied? A study of what comes next following the supreme court's historic tie on DACA and DAPA », Georgetown Immigration Law Journal, vol. 31, nº 1 , 2016, p. 153.

34. Cour suprême des États-Unis, Arizona v. United States, 567 U.S. 387 (2012).

35. Cour suprême des États-Unis, Reno v. Arab anti-discrimination Committee, 525 U.S. 471 (1999).

36. Megan L. MALLAMAS, « American animus: Where Trump v. Hawaii leaves the animus doctrine today », op. cit., p. 155-157.

37. Le Pacte international relatif aux droits civils et politiques (1966) précise que les États signataires se doivent de garantir les droits qu'il proclame à « tous les individus présents sur leur territoire et relevant de leur compétence » (art. 2\$1).

38. Tweet de Donald Trump du 19 juin 2020. URL : https://twitter.com/realDonaldTrump/status/ 1273967323901317120. Consulté le 30 août 2020.

\section{ABSTRACTS}

The Supreme Court of the United States blocked the Trump's administration decision to rescind a program called DACA, which protects about 800,000 unauthorized aliens who arrived in the United States as children from deportation. This decision, based on a violation of the Administrative Procedure Act, protects temporarily DACA recipients. However, the Court rules out questions relating to the separation of powers in immigration matters and the constitutional protection of irregular aliens.

La Cour suprême des États-Unis a bloqué la décision de retrait par l'administration Trump d'un programme appelé DACA, qui protège de l'éloignement environ 800000 étrangers en situation irrégulière arrivés aux États-Unis pendant leur enfance. Cette décision, fondée sur la méconnaissance par l'administration de la procédure administrative, protège temporairement les bénéficiaires de DACA. La Cour écarte toutefois les questions liées à la séparation des pouvoirs en matière d'immigration, et à la protection constitutionnelle des étrangers en situation irrégulière. 
AUTHOR

LÉA BOINNARD

Doctorante au Centre Universitaire de Recherches sur l'Action Publique et le Politique,

Épistémologie et Sciences Sociales (CURAPP-ESS), Université de Picardie Jules Verne. 